\title{
Optical polarization observations in the region of Stock $16^{\star}, \star \star$
}

\author{
C. Feinstein ${ }^{\star \star \star}$, G. Baume $e^{\star \star \star}$, M. M. Vergne $e^{\star \star \star}$, and R. Vázquez ${ }^{\star \star \star}$
}

\begin{abstract}
Facultad de Ciencias Astronómicas y Geofísicas, Observatorio Astronómico, Paseo del Bosque, 1900 La Plata, Argentina and Instituto de Astrofísica de La Plata, CONICET
\end{abstract}

Received 25 February 2003 / Accepted 16 July 2003

\begin{abstract}
We present ( $U B V R I)$ multicolor linear polarimetric data for 26 of the brightest stars in the area of the open cluster Stock 16 that were considered to study the properties of the ISM (interstellar medium) towards the cluster. Our data yield a mean polarization percentage of $P \sim 2.5 \%$, close to the polarization value produced by the ISM with normal efficiency $\left(P_{\lambda_{\max }} \sim 5 E_{B-V}\right)$ undergoing a color excess of $E_{B-V}=0.51$. The mean angle of the polarization vectors, $\theta=74.9$, agrees quite well with the expected angle produced by dust particles aligned in the direction of the galactic disk (and the magnetic field) in the region. A study of the extinction suffered by the stars in the zone was also performed combining our new data with previous photometric data. In this sense, our analysis indicates that the visual absorption affecting Stock 16 stars is mainly produced in front of the cluster by a dust cloud at approximately $500 \mathrm{pc}$ from the sun. The large polarization value of the nonmember star, WR 51, confirms its background star nature.
\end{abstract}

Key words. open clusters and associations: individual: Stock16

\section{Introduction}

Polarimetry is a very useful tool to get significant information (magnetic field direction, $\lambda_{\max }, P_{\max }$, etc.) from the dust located in front of a luminous object. Young open clusters are very good candidates to carry out polarimetric observations, because previous photometric and spectroscopic studies of these clusters have given detailed information on the color and luminosity of the main sequence stars of the cluster. So, we can compute the physical parameters of the clusters (age, distance, extinction, membership, etc.) and then, with the polarimetric data, we can study the location, size, and efficiency of the dust grains and the different directions of the galactic magnetic field in the line of sight to the cluster. As the open clusters are also spread within an area, we can analyze the evolution of the physical parameters all over the region.

In this framework, we are conducting systematic polarimetric observations in a large number of galactic open clusters. Following our ongoing program, we started polarimetry in the open cluster Stock 16 [C1315-623]. Located East of the Coal

Send offprint requests to: C. Feinstein, e-mail: cfeinstein@fcaglp.edu.ar

* Based on observations obtained at Complejo Astronómico El Leoncito (CASLEO), operated under agreement between the CONICET and the National Universities of La Plata, Córdoba, and San Juan, Argentina.

$\star \star$ Tables 1 and 2 are only available in electronic form at the CDS via anonymous ftp to cdsarc.u-strasbg.fr (130.79.128.5) or via http://cdsweb.u-strasbg.fr/cgi-bin/qcat?J/A+A/409/933 $\star \star \star$ Member of Carrera del Investigador Científico, CONICET, Argentina.
Sack in Crux $(l=306.11, b=+0.14)$, it is a very young and poorly populated stellar cluster at a distance of $1.9 \pm 0.08 \mathrm{kpc}$ (Turner 1985). The cluster is seen projected against the HII region RCW 75 where the star HD 115455 (LSS 3019), an O7.5III((f)) star (Walborn 1973), is probably the main source of the UV photons that excite the emission nebulae (Turner 1985).

Several photometric studies of the brightest members of Stock 16 have been carried out in the past, e.g. Lyngå (1970), Crampton (1971), Lundströn \& Stenholm (1980). In particular, Turner (1985) undertook $U B V$ photometry of 33 members, finding an age of $\sim 5 \times 10^{6}$ years, comparable to the ages he found for Cen OB1 and Cen R1 (from $\sim 10^{6}$ years to $\sim 8 \times 10^{6}$ years). He also derived a color excess $E_{B-V}=0.49$ for the cluster and a distance of $1.90 \pm 0.08 \mathrm{kpc}$, which is similar to a previous distance estimation for Cen OB1 and Cen R1 $(1.88 \pm 0.24 \mathrm{kpc})$. This suggests that Stock 16 probably belongs to a more complex large scale structure. In addition, a recent deep photometric CCD study of the cluster has shown the presence of two other distant stellar groups, also composed early type stars (Vázquez et al. 2003), with mean color excesses of $E_{B-V}=0.84$ and $E_{B-V}=1.18$ respectively. Besides, this work found a mean color excess of $E_{B-V}=0.51$ for the cluster Stock 16 and confirmed its distance.

Not far from the cluster center, northwest of it, are located the two nonmember Wolf Rayet stars, WR 50 (LSS 3013, MR44) and WR 51 (LSS 3017, MR 45). On the basis of photometric arguments, Turner (1985), van der Hutch (2001) and Vázquez (2003) indicate they are undoubtedly behind the cluster. About 7' north of Stock 16, we find the variable star cepheid V378 Cen which is a cluster nonmember object according to 
Turner (1985). Two other particular objects in this region are stars 60a and 60b (identifications from van den Bergh \& Herbst 1975), placed south-east of the cluster, at the west side end of a large dust complex below an elephant trunk structure. These stars are embedded in reflection nebulae assumed to be located on the optical boundaries of a ionization/shock front. Turner (1985) claims they are members of a new star generation, while Baume (1999) and Vázquez et al. (2003) keep them as members of Stock 16, mainly because they are at the same distance as the cluster, and their reddenings coincide with the mean cluster reddening. As already mentioned, Stock 16 seems to be an excellent candidate for a study of the interstellar medium (ISM) given its proximity with the west edge of a dense dust cloud and because it appears in the sky at the end of an elephant trunk structure.

In this paper we report the results of the first multicolor $(U B V R I)$ linear polarization observations of 26 stars in the region of Stock 16. In the next sections we will discuss observational procedures, data calibration and results in terms of both the individual stars and the cluster as a whole.

\section{Observations and data reduction}

Data on linear optical polarimetry were obtained during two observing runs at the Complejo Astronómico El Leoncito (CASLEO) in San Juan, Argentina, in 1995 (May 30 to June 3) and in 1997 (June 27-29). The observations were carried out using the Torino five-channel photopolarimeter attached to the 2.15-m telescope. Each star was observed simultaneously through the Johnson-Cousins broad band UBVRI filters $\left(\lambda_{U \mathrm{eff}}=0.360 \mu \mathrm{m}, \lambda_{B \mathrm{eff}}=0.440 \mu \mathrm{m}, \lambda_{V \mathrm{eff}}=0.530 \mu \mathrm{m}\right.$, $\left.\lambda_{\text {Reff }}=0.690 \mu \mathrm{m}, \lambda_{\text {Ieff }}=0.830 \mu \mathrm{m}\right)$. Standard stars for null polarization and for the zero point of the polarization position angle were observed several times each night for calibration purposes.

The polarimetric observations are listed in Table 1 which shows, in a self explanatory format, the stellar identification as given by Turner (1985), the polarization percentage average $\left(P_{\lambda}\right)$ and the position angle of the electric vector $\left(\theta_{\lambda}\right)$ through each filter, along with their respective mean errors computed as described by Maronna et al. (1992). Four additional stars, not observed by Turner, are indicated as A, B, C, D (see Fig. 1 for a finding chart). Since the Torino photopolarimeter collects photons simultaneously in all the filters $(U B V R I)$, the final data from each filter may be of different quality, especially those in the $U$ band. Therefore, observations whose values are above the $3 \sigma$ error level were ruled out and are not included in Table 1.

In order to detect nearby objects among the observed stars, we have searched in the Hipparcos/Tycho Catalogue. Though stars \#1, 2, 3, 4, 5, 8, 9, 10, 12 and 60a are included in this catalogue, they are so far away from the Sun that the errors of their parallax measures make them of no further utility. We notice that stars \#2, 4 and 8 were considered cluster nonmember stars according to Turner (1985). However, they also have meaningless parallaxes in Hipparcos data, and therefore their distances are probably in excess of $1 \mathrm{kpc}$.

\section{Results}

The sky projection of the $V$-band polarization vectors for the observed stars in Stock 16 are shown in Fig. 1. The dot-dashed line superimposed to the figure is the galactic parallel $b=0.08$ denoting the close alignment of the polarization vectors with the projection of the galactic plane. This indicates that the dust is aligned by a magnetic field close to the direction of the galactic disk and that the uniform distribution of the vectors suggests that the dust layer producing the polarization is located in an undisturbed place in our Galaxy, too.

Figure 2 (upper panel) shows the $P_{V}$ vs. $\theta_{V}$ diagram. The mean polarization of the stars in the cluster turns out to be $P_{V}=2.64 \%$. In principle, the Wolf Rayet star WR 51 has a polarization value higher than the rest of the stars in the sample, as expected for a star located behind the cluster at a distance of more than $8 \mathrm{kpc}$ (van der Hutch 2001; Vázquez et al 2003) with $E_{B-V}=1.39$ (Schmutz \& Vacca 1991) even assuming that part of its polarization may be intrinsic. Let us notice that the other Wolf-Rayet (WR 50) has a polarization value similar to the mean of cluster stars despite being a non member located at more than $5.5 \mathrm{kpc}$ (Conti \& Vacca 1990) or $3.6 \mathrm{kpc}$ (Vázquez et al. 2003). Specially interesting are stars 60a and $60 \mathrm{~b}$, embedded in small reflection nebulae at the south-east of Stock 16, that show polarization angles slightly larger than the mean of the cluster stars. This has been probably caused by the influence of reflection nebulae which could not be completely removed from the observations of these stars.

Figure 2 (lower panel) presents the histogram of polarization angles (excluding the data for stars 60a and 60b). By fitting the sample using a Gaussian we find a mean polarization angle of $74^{\circ} .9$ (the mean polarization angle of the galactic disk is 78.4) with a full width at half maximum, FWHM, of 6.7 Other clusters, such as NGC 6193 and NGC 6204 in ARA OB1 (Waldhausen et al. 1999) and Trumpler 27 (Feinstein et al. 2000 ), show a $F W H M$ in the order of $\sim 10^{\circ}$. Therefore, we conclude that the small value of the FWHM in the region is mainly due to the action of a single dust component as described below. Turner (1985) has successfully attempted to locate the dust layer that produces extinction. He has shown that the Cepheid star V378 Cen is too bright to be a member of the cluster though its extinction compares fairly well with that of Stock 16 . According to Turner's study, V378 Cen is located at $\sim 700 \mathrm{pc}$, thus being a foreground object. Moreover, by means of the reddening distribution of foreground objects, Turner was able to determine that extinction takes place within $500 \mathrm{pc}$ from the Sun, while the cluster is located at $1.9 \mathrm{kpc}$. In addition, Neckel \& Klare (1980) have studied the distribution of extinction values produced by the ISM in our Galaxy as a function of the distances and locations of more that 11000 stars. Taking this into account, the distance of the dust layer derived by Turner (1989) is quite compatible with the result found by Neckel \& Klare (1980) in this region. Our results are also entirely compatible with the finding of Turner (1985) because a) most of the dust appears not related with the cluster itself as the polarization vectors are aligned with the projection of the Galactic disk, and b) there is a very low dispersion of polarization angles. 


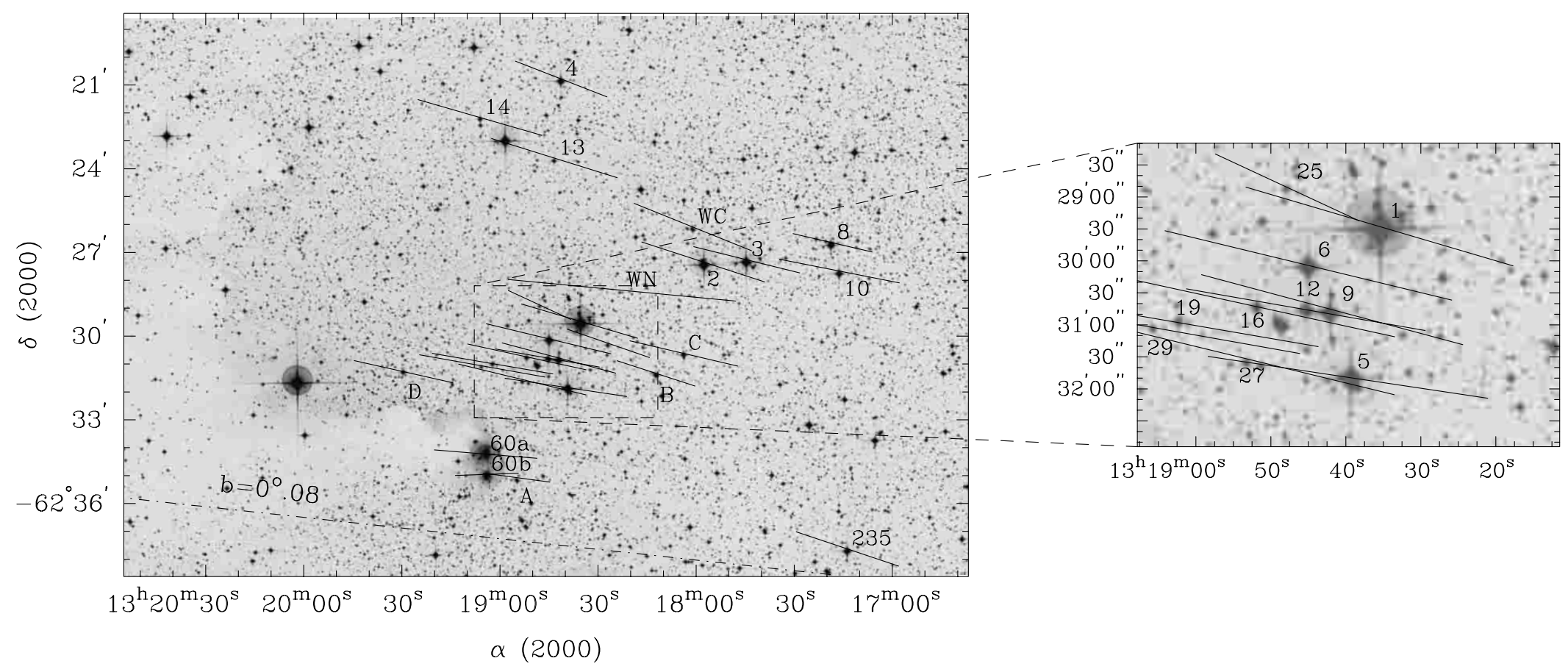

Fig. 1. Projection on the sky of the polarization vectors (Johnson $V$ filter) of the stars observed in the region of Stock 16 . The dot-dashed line is the galactic parallel $b=0.08$ 


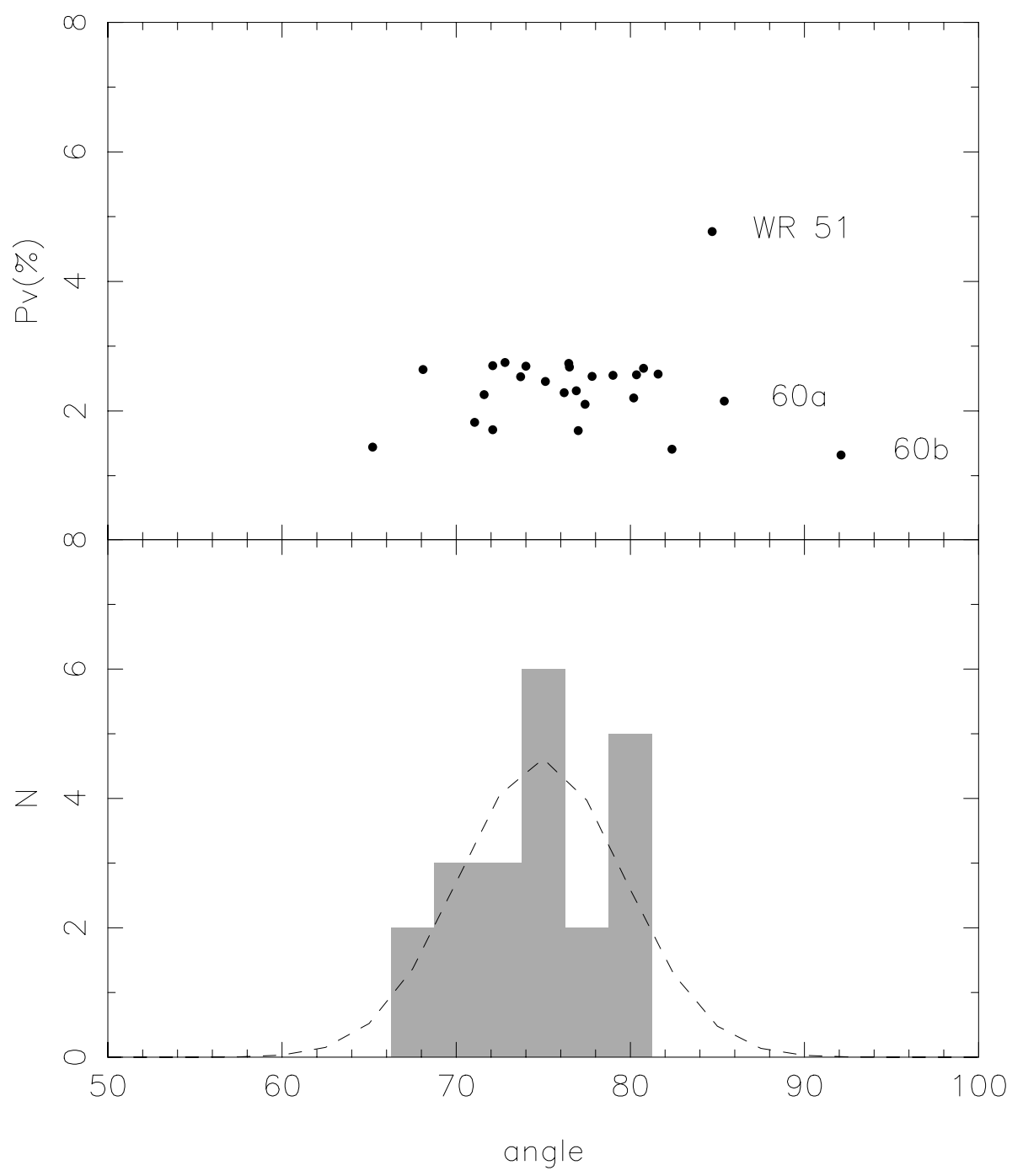

Fig. 2. Top: $V$ band polarization percentage of the stellar flux $\left(P_{V}(\%)\right)$ vs. the polarization angle $\theta_{V}$ for each star. Bottom: Histogram of the polarization angle $\left(\theta_{V}\right)$ for the observed stars. The dashed line is the Gaussian fit to the data for members of Stock 16.

Although the bulk of the extinction is clearly associated with this layer of dust in front of Stock 16, there is evidence of some intra-cluster dust component. We will discuss the existence of this intra-cluster dust in the next section.

\section{Analysis and discussion}

To analyze the data, the polarimetric observations in the five filters were fitted in each star using Serkowski's law of interstellar polarization (Serkowski 1973). That is:

$P_{\lambda} / P_{\lambda \max }=\mathrm{e}^{-K \ln ^{2}\left(\lambda_{\max } / \lambda\right)}$.

We will assume that, if polarization is produced by aligned interstellar normal dust particles, the observed data (in terms of wavelength, $U B V R I$ ) will then follow (1) and each star will have a $P_{\lambda_{\max }}$ and $\lambda_{\max }$ values.

To perform the fitting we adopted $K=1.66 \lambda_{\max }+0.01$ (Whittet et al. 1992). For each star we also computed the $\sigma_{1}$ parameter (the unit weight error of the fit) in order to quantify the departure of our data from the "theoretical curve" of Serkowski's law. In our scheme, when a star shows $\sigma_{1}>1.5$, it is indicating the presence of intrinsic stellar polarization. The $\lambda_{\max }$ values can be used to test the origin of the polarization. In fact, those objects which have $\lambda_{\max }$ lower than the average value of the interstellar medium $(0.545 \mu \mathrm{m}$, Serkowski et al. 1975) are candidates to have an intrinsic component of polarization as well (cf. Orsatti et al. 1998). Another criterion to detect intrinsic stellar polarization comes from computing the dispersion of the position angle for each star normalized by the average of the position angle errors $(\bar{\epsilon})$. The values obtained for $P_{\lambda_{\max }}$, the $\sigma_{1}$ parameter, $\lambda_{\max }$, and $\bar{\epsilon}$ together with the identification of stars are listed in Table 2.

The $P_{\lambda}$ and $\theta_{\lambda}$ values in those stars which are candidates to have an intrinsic component of polarization (\#18, 19 and 20) are shown in Fig. 3. For comparison purposes, the best Serkowski's law fit has been plotted in each case using a continuous line.

Figure 4 shows the histogram for all the observed $\lambda_{\max }$ values. As this figure shows, the most probable value of $\lambda_{\max }$ for stars in Stock 16 is around $0.545 \mu \mathrm{m}$ in agreement with the average value of the galactic ISM found by Serkowski et al. (1975). 


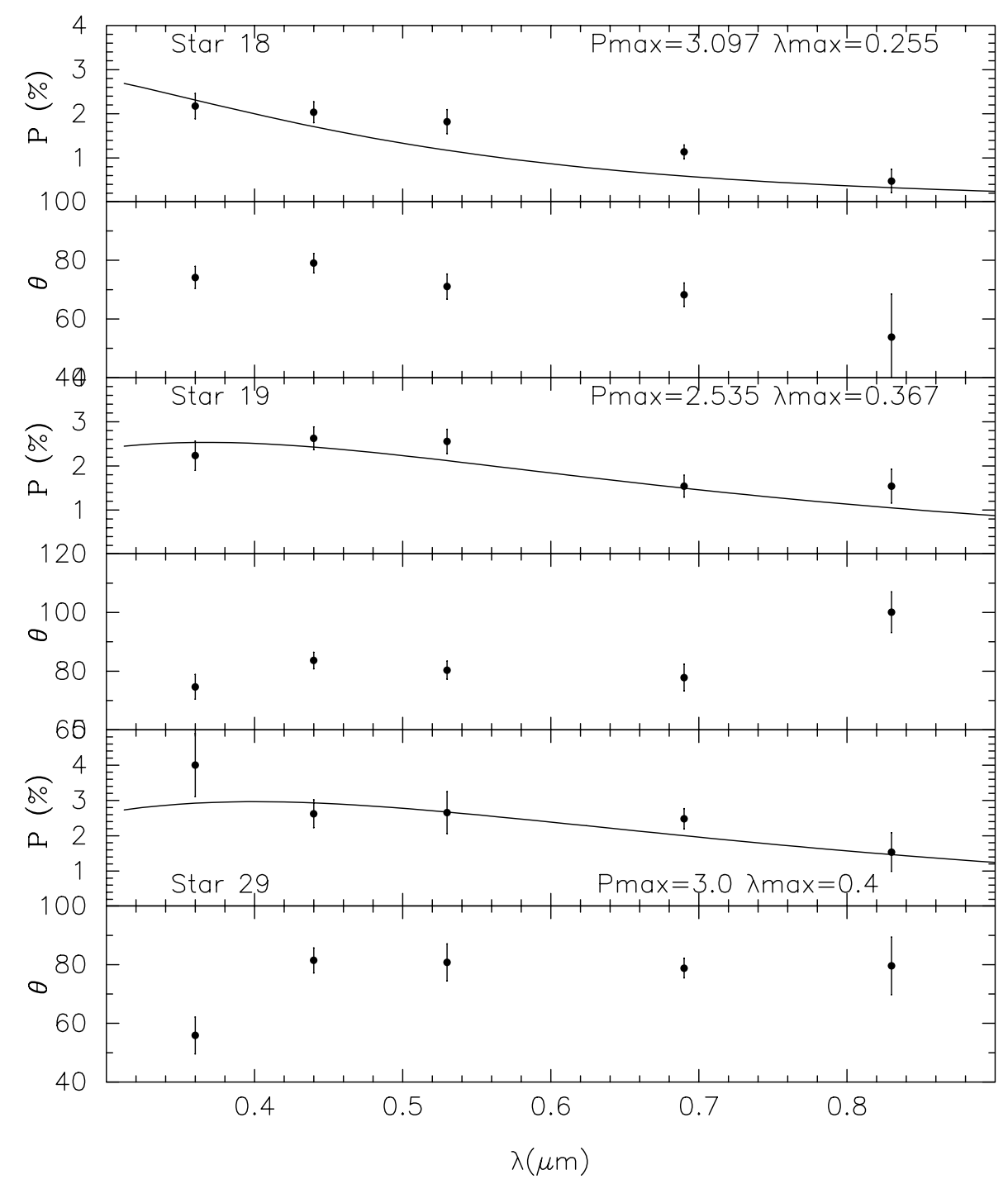

Fig. 3. Plot of the observed $P_{\lambda}(\%)$ and $\theta_{\lambda}$ data for objects showing large departures from the Serkowski law, the solid line is the best fit.

The polarization efficiency of the dust towards Stock 16 is estimated from the $P_{\lambda_{\max }}$ vs. $E_{B-V}$ individual plots. Individual color excesses $E_{B-V}$ of a few stars having unique reddening solution in the color-color diagram (Baume 1999; Vázquez et al. 2003) are plotted in Fig. 5. Assuming normal interstellar material characterized by $R=3.2$, the empirical upper limit relation for the polarization efficiency given by $P_{\lambda \max }=R A_{v} \sim 9 E_{B-V}$ (Serkowski et al. 1975) is depicted by the continuous line in this figure. Indeed, this line represents the maximum efficiency of polarization produced by the interstellar dust. Likewise, the dashed line $P_{\lambda_{\max }} / E_{B-V} \sim 5$, also shown in Fig. 5, represents the observed normal efficiency of the polarizing properties of the dust (Serkowski et al. 1975). In the particular case of the open cluster Stock 16, the figure indicates that the polarization efficiency appears lower than the average observed in our Galaxy.

The present polarimetric observations are therefore (as shown in the previous section) entirely compatible with a dust distribution model where most of the extinction is produced within $500 \mathrm{pc}$ from the Sun as indicated by Turner (1985).
However, there is some evidence of an intra-cluster dust component inside Stock 16. Turner (1985) has found that five out of his nine faintest cluster members lie well above the ZAMS. On this basis, he argued that the location of those stars above the ZAMS is due to their binary nature. However, another explanation for the observed pattern in the color-color diagram is that the main sequence of Stock 16 is slightly broadened due to the presence of dust inside the cluster producing different amounts of stellar reddenings (Baume 1999; Vázquez et al. 2003). These authors have shown that a group of stars near the cluster center show $E_{B-V}$ color excesses 0.1 more reddened than the other cluster stars. In this context, we favor the hypothesis of a main dust component located between the cluster and the Sun (hereafter we will call this layer the "first component"), while a second minor component is "inside" the cluster. This is illustrated in Fig. 5, which shows that the stars observed in the present work split into two groups (one 0.1 mag more reddened than the other) as found by Baume (1999) and Vázquez et al. (2003). The less reddened group represented in the figure is composed by stars \#12,16, 19 and the more reddened one, by stars \#1, 5, 6, 9, 18, 60a and 60b. It seems that the first less 


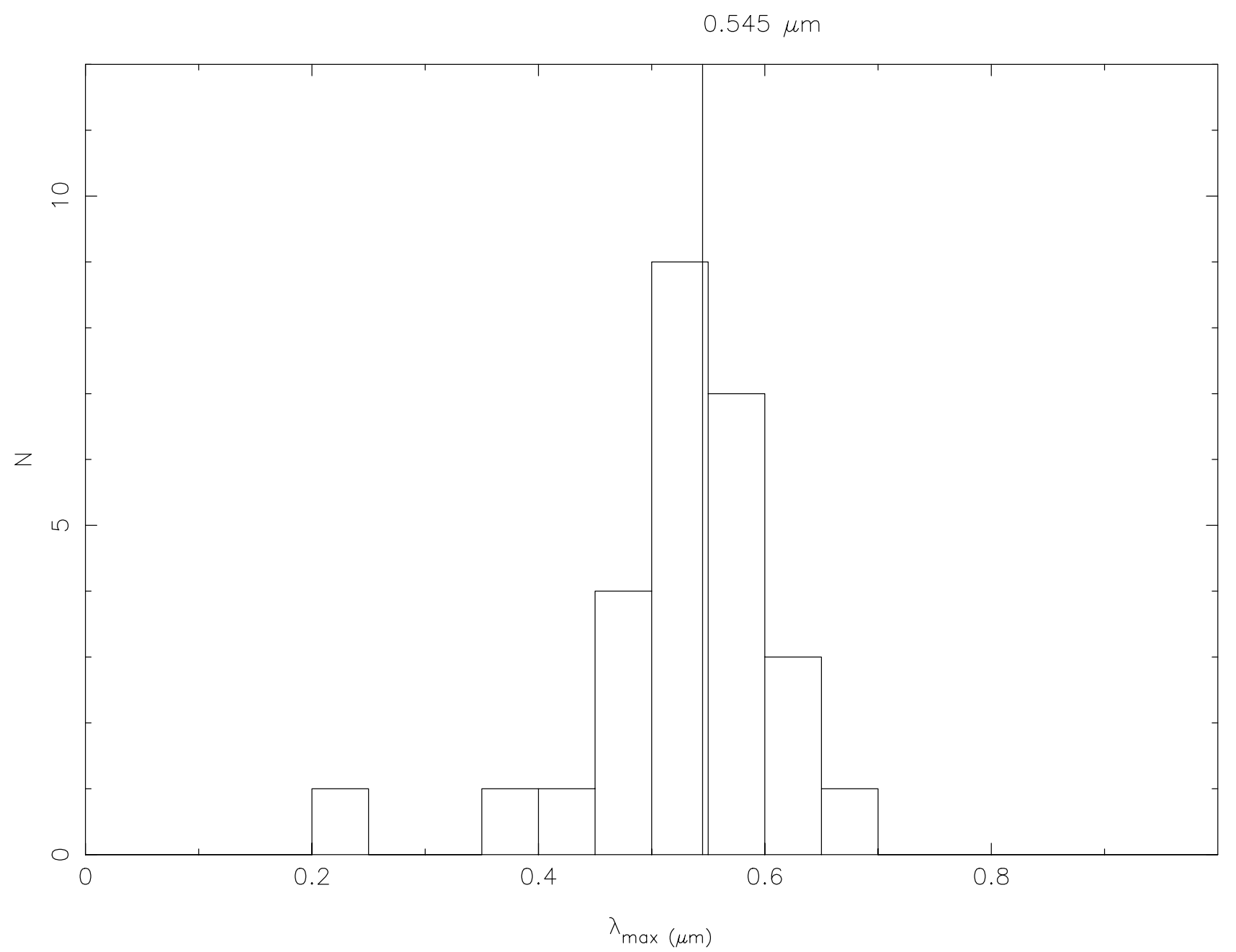

Fig. 4. Histogram of the parameter $\lambda_{\max }$ obtained fitting the Serkowski law to the observations.

reddened star group is very likely composed by "front-side" stars not suffering from internal extinction. That is, the $E_{B-V}$ of this group of stars is representative of the first component of dust (located between the Sun and the cluster).

To attempt isolating the second polarization component we should subtract the interstellar polarization $(I P)$ from our observations. The problem to do that in this case is that the intracluster component only produces a very low $E_{B-V}$ value of $0.1 \mathrm{mag}$ (Vázquez et al 2003). At the level of the errors associated to our observations, the intracluster material causing a value $E_{B-V}=0.1$ yields polarization values comparable to the typical errors of the individual observations. Therefore, it becomes useless to try isolating it.

From Fig. 5 it can also be inferred that this second component of the extinction does not produce an increment in the percentage of the polarization as expected, should this component be aligned with the projection of the galactic disk. Therefore, it might be possible for this intra-cluster component to have a polarization angle different from that of the IP component, thus depolarizing the starlight. However, that is very unlikely. In fact, the polarization angles of this subgroup of reddened stars do not seem to be different, as would have been the case if another polarization component with a different polarization angle was added. Moreover, the very narrow distribution of the polarization angles in the direction of the Galactic disk $\left(\mathrm{PA}=78^{\circ} .4\right)$ shown in Fig. 2 (lower panel) indicates that it is very unlikely we are observing an angle distribution resulting from a combination of several dust layers each of them polarizing the starlight in different directions.

\section{Summary}

For the first time linear multicolor polarization observations for a sample of 26 stars in the region of Stock 16 is presented. Our observations are compatible with Turner's suggestions (1985) that the bulk of the extinction in this direction is produced in a dust cloud within $\sim 500 \mathrm{pc}$ from the Sun. The observed values of the polarization are compatible with the expected extinction $E_{B-V}=0.51$ (Baume 1999; Vázquez et al. 2003) and the angles of the polarization vectors are also the expected ones for the direction of the Galactic disk in the region. We found that the distribution of angles of the polarizations vectors is narrower $(F W H M=6.7)$ than the typical value found for other clusters $F W H M \sim 10^{\circ}$, as in Trumpler 27 (Feinstein et al. 2000) and in NGC 6193 and NGC 6204 in ARA OB1 (Waldhausen et al. 1999). There is photometric evidence for the existence of some intracluster dust (Turner 1985; Baume 1999; 


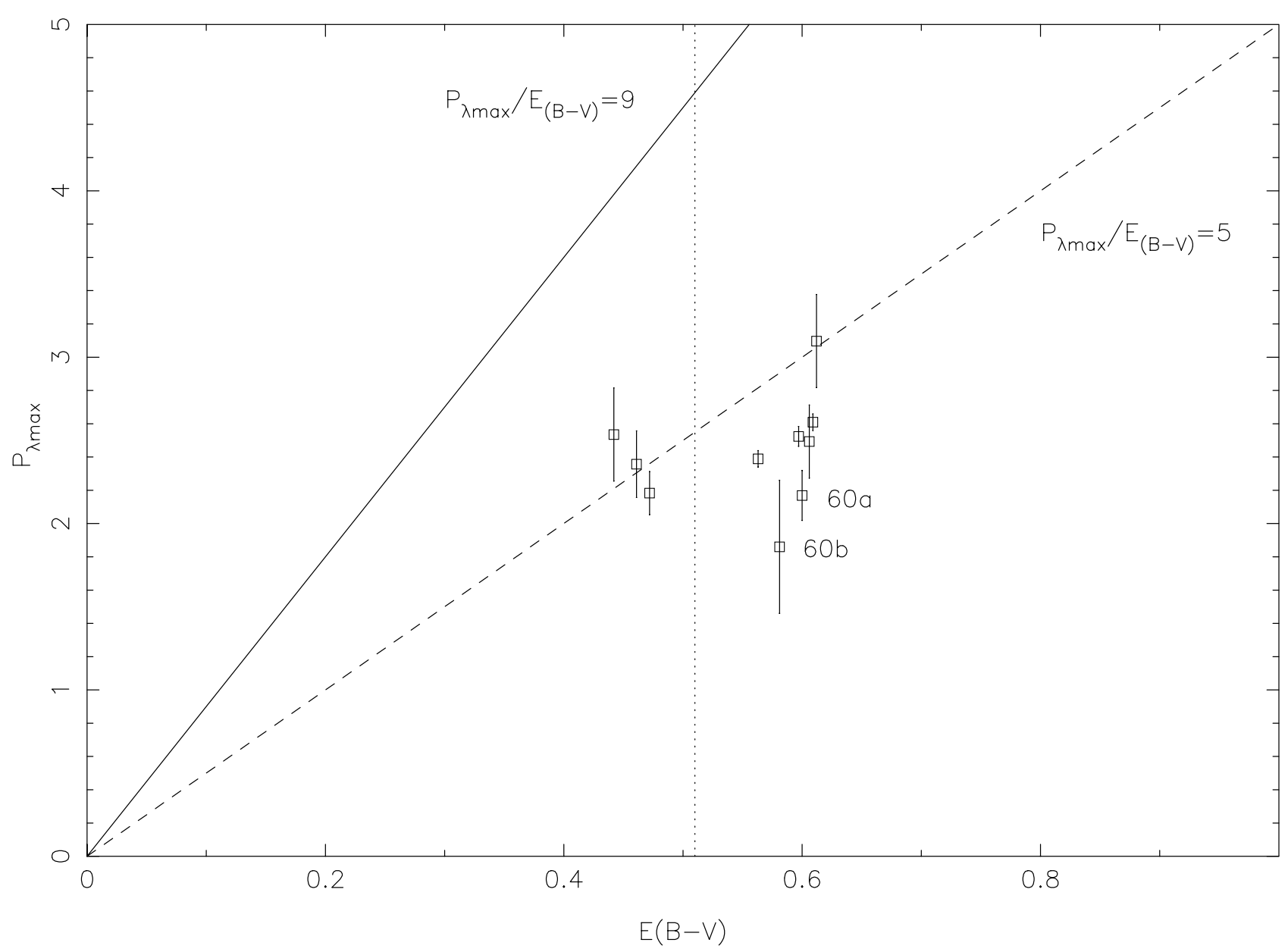

Fig. 5. Plot $P_{\lambda \max }$ vs. $E_{B-V}$ for stars of Stock 16. The solid line is $P_{\lambda \max }=9 E_{B-V}$, and the dashed line is $P_{\lambda \max }=5 E_{B-V}$. The vertical dotted line is the mean cluster extinction, $E_{B-V}=0.51$.

Vázquez et al. 2003) and we do not rule out a contribution to the observed polarimetry from this component but it probably takes place at the level of the errors of our data.

Acknowledgements. We wish to acknowledge the technical support at CASLEO during the observing runs. We also acknowledge the use of the Torino Photopolarimeter built at Osservatorio Astronomico di Torino (Italy) and operated under agreement between Complejo Astronómico El Leoncito and Osservatorio Astronomico di Torino. Special thanks go to Ana M. Orsatti and Virpi Niemela for their valuable comments.

\section{References}

Baume, G., 1999 Ph.D. Thesis, Facultad de Ciencias Astronómicas y Goefísicas, Universidad Nacional de La Plata, Library Conti, P. S., \& Vacca, W. D. 1990, AJ, 100,431

Crampton, D. 1971, AJ, 76, 260

Feinstein, C., Baume, G., Vazquez, R., Niemela, V., \& Cerruti, M. G. 2000, AJ, 120, 1906

Lundströn, I., \& Stenholm, B. 1980, Repts. Obs. Lund, No. 16
Lyngå, G. 1970, in The Spiral Structure of Our Galaxy, ed. W. Becker \& G. Contopoulous (Dordrecht: Reidel), IAU Symp, 38, 177

Neckel, Th., \& Klare, G. 1980, A\&AS, 42, 251

Orsatti, A. M., Vega, E., \& Marraco, H. G. 1998, AJ, 116, 226

Serkowski, K. 1973, in Interstellar Dust and Related Topics, ed. J. M. Greenberg, \& H. C. van de Hults (Dordrecht: Reidel), IAU Symp., 52,145

Serkowski, K., Mathewson, D. L., \& Ford, V. L. 1975, ApJ, 196, 261

Smith, L. F., Shara, M. M., \& Moffat, A. F. J. 1990, ApJ, 358, 229

Smith, L. F., Shara, M. M., \& Moffat, A. F. J. 1996, MNRAS, 281, 163

Schmutz, W., \& Vacca, W. D. 1991, A\&AS, 89, 259

Turner, D. G. 1985, ApJ, 292, 148

van den Bergh, S., \& Herbst W. 1975, AJ, 80, 208

van der Hutch, K. A. 2001, New Astron. Rev., 45, 135

Vázquez, R., Baume, G., Feinstein, C., Núñez, J., \& Vergne, M. M. 2003, in preparation

Walborn, N. R. 1973, AJ, 78, 1067

Waldhausen, S., Martínez R., \& Feinstein, C. 1999, AJ, 117, 2882

Whittet, D. C. B., Martin, P. G., Hough, J. H., Rouse, M. F., Nailey, J. A., \& Axon, D. J. 1992, ApJ, 386, 562 